\title{
MicroRNA-539 inhibits the proliferation and migration of gastric cancer cells by targeting SRY-box 5 gene
}

\author{
SHI DING and YANPING ZHANG \\ Department of Medicine, Changde Vocational Technical College, Changde, Hunan 415000, P.R. China
}

Received February 7, 2018; Accepted October 8, 2018

DOI: $10.3892 / \mathrm{mmr} .2019 .10486$

\begin{abstract}
The aim of the present study was to investigate the effect of microRNA (miR)-539 on the proliferation and migration of gastric cancer cells, and explore the underlying mechanism. Gastric cancer cell lines with high or low miR-539 and SRY-box 5 (SOX5) expression levels were constructed by transfection. The proliferation of gastric cancer cells was then detected by Cell Counting Kit- 8 assay and cell migration was tested by transwell assay. The results revealed low expression of miR-539 and high expression of SOX5 in gastric cancer tissues and cells as compared with the levels in normal tissues and cells, suggesting that there was a negative correlation between miR-539 and SOX5. Dual-luciferase reporter experiments demonstrated that miR-539 directly targeted SOX5. The proliferation and migration of gastric cancer cells were negatively regulated by the overexpression of miR-539, while positively regulated by the overexpression of SOX5. Notably, SOX5 overexpression attenuated the inhibitory effect of miR-539 on gastric cancer cells. The results suggested that SOX5 is a target gene of miR-539, and that miR-539 inhibits the proliferation and migration of gastric cancer cells by targeting SOX5.
\end{abstract}

\section{Introduction}

Gastric cancer is one of the most common malignant tumors in humans. Despite its morbidity and mortality exhibiting a steady downward trend worldwide, gastric cancer remains a great burden to human health as the third leading cause of cancer-associated mortality $(1,2)$. A report revealed that the region with the highest incidence of gastric cancer is East Asia, particularly China, with an annual incidence of approximately 4-6 cases per 10,000 individuals (3). In addition, the National Cancer Institute data indicated that the incidence and diagnosis

Correspondence to: Dr Yanping Zhang, Department of Medicine, Changde Vocational Technical College, 4253 Renmin Road, Wulin, Changde, Hunan 415000, P.R. China

E-mail: zhangyanping_0919@163.com

Key words: gastric cancer, microRNA-539, SRY-box 5, proliferation, migration of this disease is mainly between the ages of 60-70 years, and the majority of patients have a poor prognosis due to diagnosis in the late stages of the disease (4). Approximately $90 \%$ of stomach tumors are adenocarcinomas, which are classified into two main histologic types: High differentiated or intestinal-type gastric cancer; and undifferentiated or diffuse-type gastric cancer. Intestinal-type gastric cancer is pertinent to atrophy of the gastric mucosa and intestinal metaplasia, while diffuse-type tumors usually arise in tissue without atrophic total gastritis (5).

MicroRNA (miR), as a type of non-encoding RNA with a length of approximately $22 \mathrm{bp}$, regulates gene expression at the post-transcriptional level. The known miRNAs regulate approximately $30 \%$ of the genes in the human genome, which participate in human development, cell proliferation and differentiation, hematopoiesis and cell apoptosis (6). Nearly $50 \%$ of the miRNAs are positioned on the tumor-associated genomic regions, including fragile sites, chromosomal amplification and loss of heterozygosity chromosome region $(7,8)$. Thus, miRNAs can significantly affect the entire process from tumor occurrence to tumor metastasis. Abnormal miRNA expression is also associated with the occurrence and development of gastric cancer, and can regulate the expression of multiple oncogenes or anti-oncogene, thereby influencing tumor cell proliferation, migration, invasion, apoptosis and other biological characteristics (9).

Low expression of miR-539 has been reported in osteosarcoma tissues, and this was negatively correlated with the clinical stage, recurrence and metastasis of the disease; thus, low expression of miR-539 may be an independent prognostic indicator of overall survival in patients with osteosarcoma (10). In hepatocellular carcinoma (HCC), miR-539 was reported to function as a tumor suppressor, and its expression was downregulated in HCC tissues and cell lines (11). However, forced expression of miR-539 was able to induce the apoptosis of HepG2 cells and enhance the sensitivity of HCC cells to arsenic trioxide (11). In nasopharyngeal carcinoma (NPC), miR-539 targeted cyclin-dependent kinase 4 to induce cell cycle arrest, resulting in the inhibition of NPC cell growth (12). In non-small cell lung cancer (13) and thyroid cancer, miR-539 inhibited the proliferation, migration and invasion of cancer cells by targeting CARMA1 (14). Although an increasing number of studies have reported that the role of miR-539 in cancer is mainly as a tumor suppressor, the specific role of miR-539 in gastric cancer remains unknown. 
Therefore, the present study focused on the role of miR-539 in the process of gastric cancer. Initially, it was demonstrated that miR-539 was poorly expressed in gastric cancer tissues and cell lines. Subsequently, it was observed that miR-539 inhibited the proliferation and migration of gastric cancer cells, and this effect may be achieved partly by targeting SRY-box 5 (SOX5). Overall, the study reveals that miR-539 serves an important role in the process of gastric cancer, indicating its potential application in the treatment of gastric cancer.

\section{Materials and methods}

Collection of clinical tissue samples. Between August 2015 and July 2016, 30 gastric cancer patients receiving surgical treatment were recruited into the present study from The First People's Hospital of Changde City (Changde, China). The inclusion criteria were as follows: Gastric cancer was confirmed with pathologic diagnosis (15); and the patients had not undergone any radiotherapy and chemotherapy prior to surgical treatment. Tumor and adjacent tissue samples were collected from the patients and stored at $-80^{\circ} \mathrm{C}$. The proportion of malignant cells in the cancer tissue samples was $>80 \%$, while the adjacent tissue samples included $<10 \%$ of malignant cells. The study was approved by the Ethics Committee of Changde Vocational Technical College Research (Changde, China). Patients provided signed informed consent prior to participation in the study.

Cell lines and cell culture. Gastric cancer cell lines SGC7901, MGC803 and HGC-27, as well as the normal gastric mucosa cell line GES-1, were purchased from American Type Culture Collection (Manassas, VA, USA) and preserved until cell recovery. The cells were cultured in $5 \% \mathrm{CO}_{2}$ at $37^{\circ} \mathrm{C}$ with Dulbecco's modified Eagle's medium (Gibco; Thermo Fisher Scientific, Inc., Waltham, MA, USA) containing $10 \%$ fetal bovine serum (FBS), penicillin $(100 \mathrm{U} / \mathrm{ml})$ and streptomycin $(100 \mu \mathrm{g} / \mathrm{ml})$.

Cell transfection. At 1 day before cell transfection, cells in the logarithmic growth phase were selected and seeded into a 96-well plate $\left(5 \times 10^{3}\right)$. miR-539 mimic, miR-539 inhibitor and scramble mimic negative control (miR-NC) were synthesized by GenePharma Co., Ltd., (Shanghai, China). The cells were transfected with $20 \mathrm{nmol} / 1 \mathrm{miR}-539 \mathrm{mimic}$ or miR-NC, and the miR-539 inhibitor (high concentration solution, $20 \mu \mathrm{m}$; diluted in $\mathrm{ddH}_{2} \mathrm{O}$ ) using Lipofectamine ${ }^{\circledR} 2000$ (Invitrogen; Thermo Fisher Scientific, Inc.) according to the manufacturer's protocol. For the rescue experiments, miR-539 mimics and SOX5 overexpression with miR-539 mimics were transfected into SGC7901 and MGC803 cells.

RNA extraction and quantitative polymerase chain reaction $(q P C R)$. Total RNA was extracted from the tissues or cultured cells using TRIzol reagent (Invitrogen; Thermo Fisher Scientific, Inc.) and a miRNeasy Mini kit (Qiagen $\mathrm{GmbH}$, Hilden, Germany) according to the manufacturers' protocol. TaqMan MicroRNA Assay (Invitrogen; Thermo Fisher Scientific, Inc.) was used to perform qPCR in order to qualify miRNA expression. RNA was converted into complementary DNA by reverse transcription using mRNA/miRNA reverse transcription kits (for mRNA, RevertAid RT Reverse
Table I. Primers used in quantitative polymerase chain reaction.

\begin{tabular}{ll}
\hline Gene & \multicolumn{1}{c}{ Primer sequences (5'-3') } \\
\hline miR-539 sense & GGAGAAATTATCCTTGGTGTG \\
U6 sense & ATTGGAACGATACAGAGAAGATT \\
SOX5 forward & AGCATGCTTACTGACCCTGATTTA \\
SOX5 reverse & GGGAGTCCTATGGCCACAAGTCT \\
GAPDH forward & TCATGGGTGTGAACCATGAGAA \\
GAPDH reverse & GGCATGGACTGTGGTCATGAG
\end{tabular}

miR, microRNA; SOX5, SRY-box 5.

Transcription kit; Fermentas; Thermo Fisher Scientific, Inc.; and for miRNA, All-in-One ${ }^{\mathrm{TM}}$ qRT-PCR Detection kit; Fumeng, Guangzhou, China), with the following temperature protocols: For mRNA, $42^{\circ} \mathrm{C}$ for $1 \mathrm{~h}$ and $70^{\circ} \mathrm{C}$ for $10 \mathrm{~min}$; and for miRNA, $37^{\circ} \mathrm{C}$ for $1 \mathrm{~h}$ and $85^{\circ} \mathrm{C}$ for $5 \mathrm{~min}$. Then qPCR was subsequently performed using SYBR Premix DimerEraser (Takara Biotechnology Co., Ltd., Dalian, China) on a 7900HT Fast Real-Time PCR system (Thermo Fisher Scientific, Inc.). The qPCR thermal cycling conditions were as follows: $95^{\circ} \mathrm{C}$ for $15 \mathrm{sec} ; 40$ cycles at $95^{\circ} \mathrm{C}$ for $5 \mathrm{sec} ; 55^{\circ} \mathrm{C}$ for $34 \mathrm{sec} ; 72^{\circ} \mathrm{C}$ for $30 \mathrm{sec}$; followed by melting curve analysis. The level of mature miR-539 was normalized to that of the U6 endogenous control, while glyceraldehyde-3-phosphate dehydrogenase (GAPDH) was used as an internal control for the detection of SOX mRNA expression. Fold changes were calculated using the comparative threshold cycle value $\left(2^{-\Delta \Delta \mathrm{Cq}}\right)$ method (16). The experiments were performed in triplicate, and the primers used are listed in Table I.

Cell proliferation test. Cells were seeded into 96-well plates at a density of 2,000 cells per well and cultured for 1,2,3,4 and 5 days after transfection. In order to determine cell proliferation, Cell Counting Kit-8 (CCK-8; Sangon Biotech Co., Ltd., Shanghai, China) assay was performed. Cells were incubated with $10 \% \mathrm{CCK}-8$ reagent at $37^{\circ} \mathrm{C}$ until visual color conversion occurred. Subsequently, the absorbance at $450 \mathrm{~nm}$ was measured using a microplate reader (Thermo Fisher Scientific, Inc.). All experiments were conducted in triplicate.

Transwell assay. Transwell assay was performed to detect cell migration and invasion. A total of $1.0 \times 10^{5}$ cells were suspended in serum-free medium and plated in the upper chambers of transwell inserts in a 6-well plate (Corning Incorporated, Corning, NY, USA). Medium containing 10\% FBS was added to the lower chamber, serving as a chemoattractant. Following incubation for $24 \mathrm{~h}$ at $37^{\circ} \mathrm{C}$ in $5 \% \mathrm{CO}_{2}$, cells in the lower chamber were fixed in methanol for $15 \mathrm{~min}$ and stained with $0.05 \%$ crystal violet in PBS for 15 min before counting under a microscope (Olympus Corporation, Tokyo, Japan). Cells that had not migrated from the upper chamber were removed by wiping the surface with a cotton swab, and invasive cells were fixed with $4 \%$ formaldehyde in PBS and subsequently stained with $1 \%$ crystal violet in $2 \%$ ethanol. Images of the cells on the lower surface of the filter were obtained under a light microscope (magnification, $\mathrm{x} 200$ ). 
A

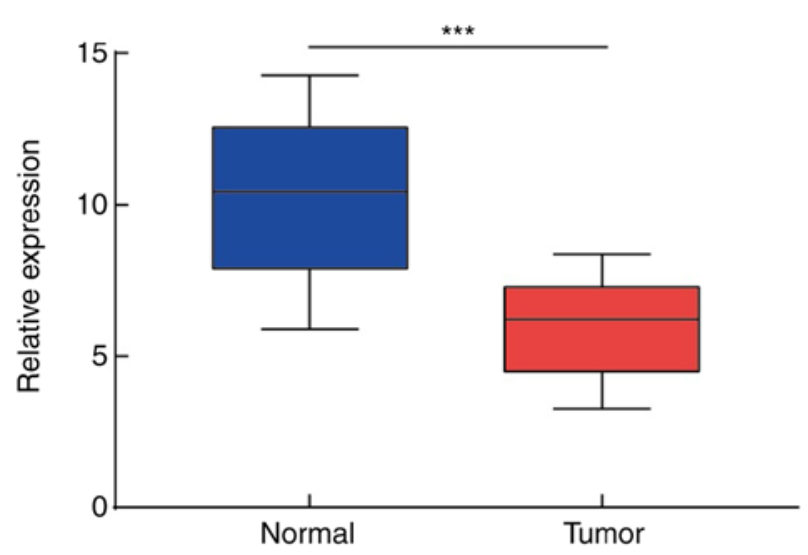

B Expression of miR-539 in cell lines

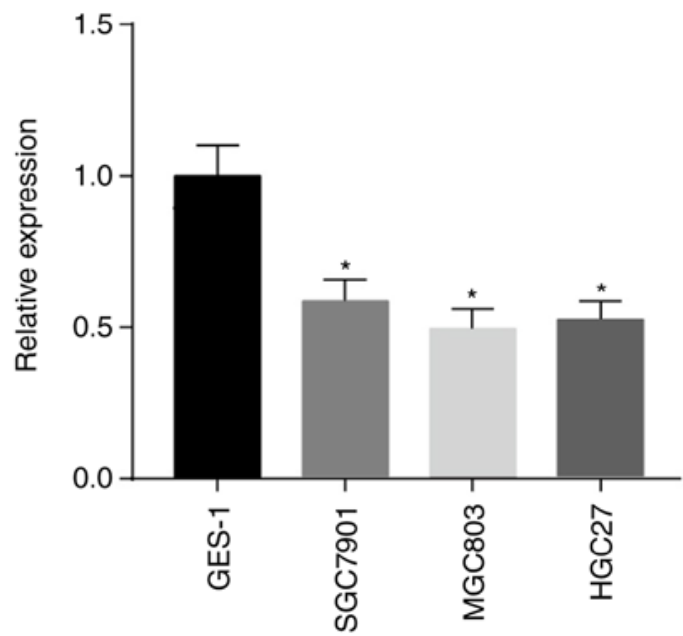

Figure 1. Expression of miR-539 in tissues and cell lines of gastric cancer. (A) Expression of miR-539 in tissues samples. The tumor and adjacent normal tissue samples were obtained from 30 gastric cancer patients receiving surgical treatment. ${ }^{* * *} \mathrm{P}<0.001$, as indicated. (B) Expression of miR-539 in gastric cancer cell lines. ${ }^{*} \mathrm{P}<0.05$ vs. normal GES- 1 cells. miR, microRNA.

Dual-luciferase reporter assay. TargetScan (www.targetscan. org/) and miRanda (www.microrna.org/microrna/home.do) were initially used to predict the potential target gene of miR-539, which was revealed to be SOX5; a luciferase assay was performed to confirm this. A fragment of the human SOX5 3'-untranslated region (3'UTR) containing the miR-539 binding site was amplified by PCR and cloned downstream of the firefly luciferase gene in the pMIR-REPORT vector (Ambion; Thermo Fisher Scientific, Inc.) to obtain the wild-type (WT) SOX5 reporter vector. The mutation fragment was cloned into the pMIR-REPORT vector to produce the pMIR-SOX5-mut (Mut) 3'UTR vector. Cells $\left(2 \times 10^{4}\right)$ were seeded into 24 -well plates on the day before transfection, and then cotransfected with the constructs and miR-539 mimic or miR-NC. Luciferase activity was measured after $48 \mathrm{~h}$ using the Dual-Luciferase Reporter Assay system (Promega Corporation, Madison, WI, USA).

Statistical analysis. The quantitative data between groups were compared and analyzed by Student's t-test (two tailed) or by one-way analysis of variance followed by Tukey's test. Data are expressed as the mean \pm standard deviation. $\mathrm{P}<0.05$ was considered to indicate a statistically significant difference.

\section{Results}

Expression of miR-539 in gastric cancer. miR-539 expression in gastric cancer cell lines, gastric mucosa cell line GES-1, and tumor and adjacent tissue samples from 30 cases gastric cancer patients was determined by qPCR. The results demonstrated that miR-539 exhibited a significantly lower expression in the gastric tumor tissue samples compared with that in the adjacent tissue samples $(\mathrm{P}<0.001$; Fig. 1A). Furthermore, in gastric cancer cell lines SGC7901, MGC803 and HGC-27, the expression of miR-539 was markedly reduced as compared with that in the gastric mucosa cell line GES-1 $(\mathrm{P}<0.05$; Fig. 1B).
Effect of miR-539 on the proliferation and migration of gastric cancer cells. The results of CCK-8 assay demonstrated that, compared with the cells in the scramble group, the cell proliferation was significantly decreased from day 2 of transfection with miR-539 mimic in the gastric cancer cell lines MGC803 (Fig. 2A and B) and SGC7901 (Fig. 2C and D). By contrast, cell proliferation was significantly promoted after transfection with miR-539 inhibitor. In the transwell assay, gastric cancer cell lines transfected with miR-539 mimic had significantly decreased migration compared with the control group, which was contrary to the enhanced migration observed in cells transfected with miR-539 inhibitor $(\mathrm{P}<0.05$ and $\mathrm{P}<0.01$; Fig. $2 \mathrm{E}$ and $\mathrm{F})$.

Targeting association between miR-539 and SOX5 gene. The association between miR-539 and SOX5 gene was verified in gastric cancer cells using a luciferase assay. The results suggested that, compared with the SOX5 Mut group and the control group, the luciferase activity of SOX5 WT reporter vector was significantly reduced in MGC803 cells transfected with miR-539 mimic, indicating that miR-539 directly targets SOX5 gene (Fig. 3A). In addition, the results of $\mathrm{qPCR}$ revealed that SOX5 expression in gastric tumor tissues was markedly higher in comparison with that in adjacent tissues (Fig. 3B). Spearman's correlation analysis further demonstrated that there was a negative correlation between miR-539 and SOX5 gene expression levels in clinical tissue samples of gastric cancer ( $\mathrm{R}=-0.76 ; \mathrm{P}<0.001$; Fig. 3C).

SOX 5 regulates the proliferation and metastasis of gastric cancer cells. CCK-8 assay demonstrated that, compared with the cells transfected with empty vector, MGC803 cells with SOX5 overexpression exhibited significantly increased proliferation $(\mathrm{P}<0.05)$; while MGC803 cells with SOX5 inhibition had notably decreased proliferation ( $\mathrm{P}<0.05$; Fig. $4 \mathrm{~A}$ and $\mathrm{B})$. The same results were confirmed in SGC7901 cells (Fig. 4C and D). In the transwell experiment, compared with the control group, the migration of MGC803 cells was significantly higher following SOX5 
A

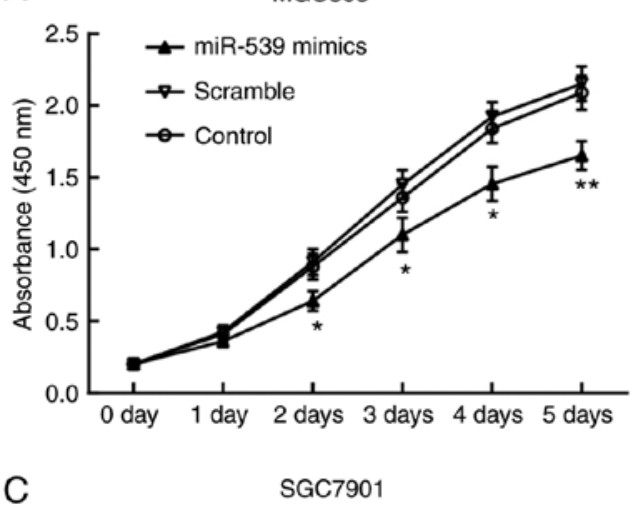

C

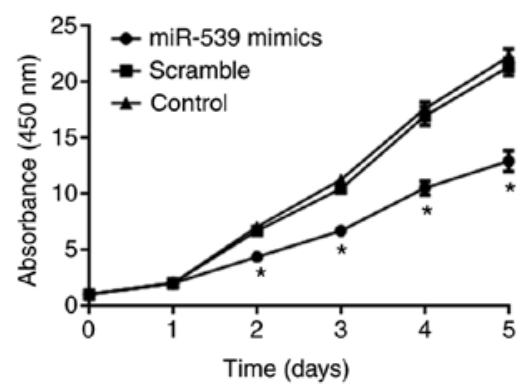

E
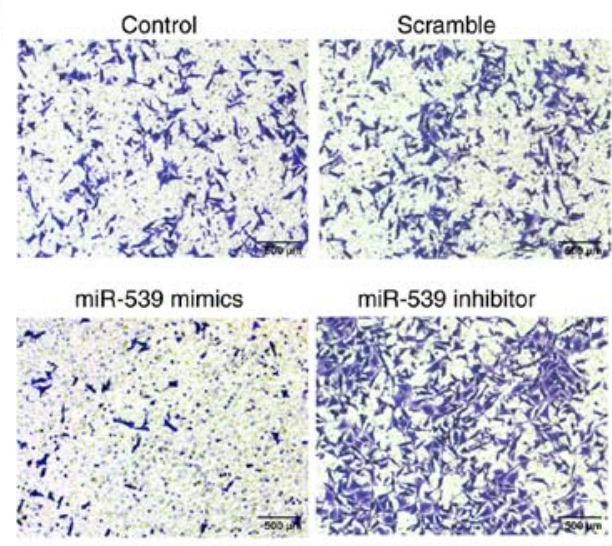

$\mathrm{F}$

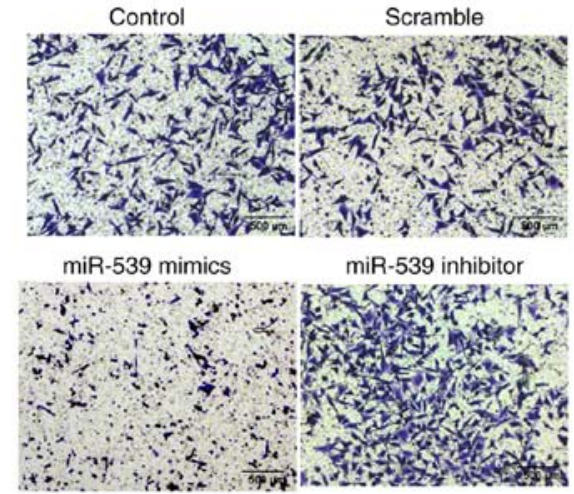

B

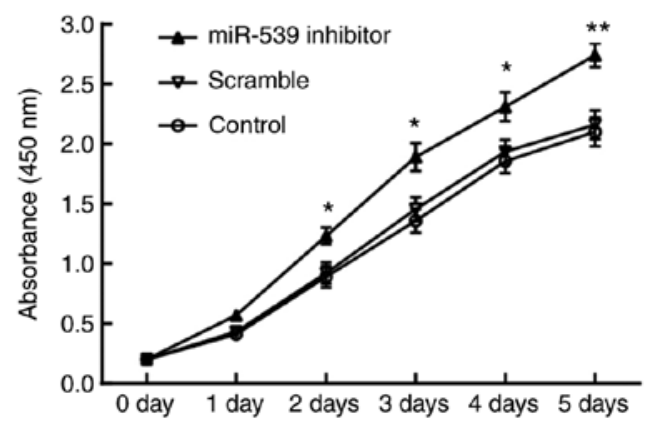

D

SGC7901
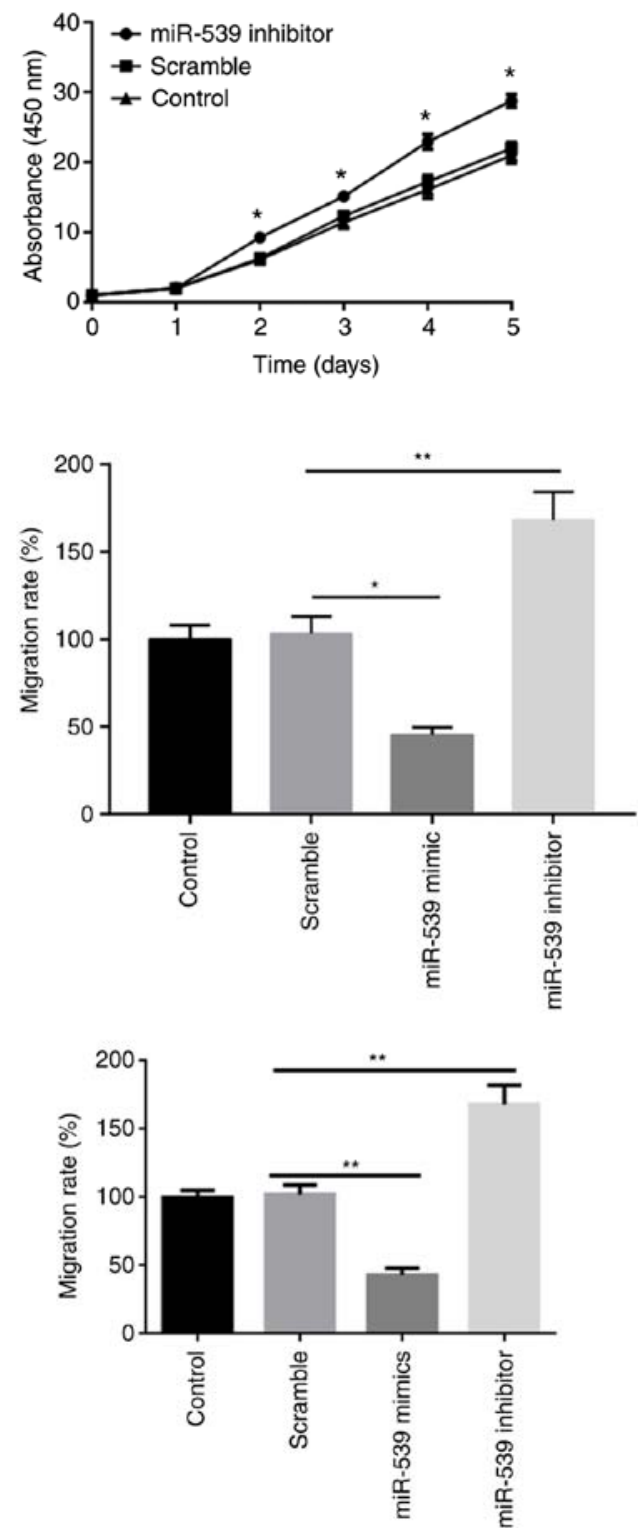

Figure 2. Effect of miR-539 on the proliferation and migration of MGC803 and SGC7901 cells. (A) miR-539 mimic or (B) inhibitor effects on the growth of MGC803 cells, and (C) miR-539 mimic or (D) inhibitor effects on the growth of SGC-7901 cells were detected via Cell Counting Kit-8 assay. "P<0.05 and ${ }^{* *} \mathrm{P}<0.01$ vs. control group. Effect of miR-539 mimic or inhibitor on the migration of (E) MGC803 and (F) SGC7901 cells was examined by transwell assays (magnification, $\mathrm{x} 200$ ). ${ }^{*} \mathrm{P}<0.05$ and $^{* *} \mathrm{P}<0.01$, as indicated. miR, microRNA.

overexpression, while it was significant lower subsequent to SOX5 inhibition (Fig. 4E-H). The same results were confirmed in SGC7901 cells (Fig. 4I and J). These results suggest that SOX5 is also involved in cell proliferation and migration in gastric cancer.
SOX5 restores the effect of miR-539 on the proliferation and migration of gastric cancer cells. The aforementioned results suggested that the proliferation and migration of gastric cancer cells were inhibited by miR-539 and promoted by SOX 5 

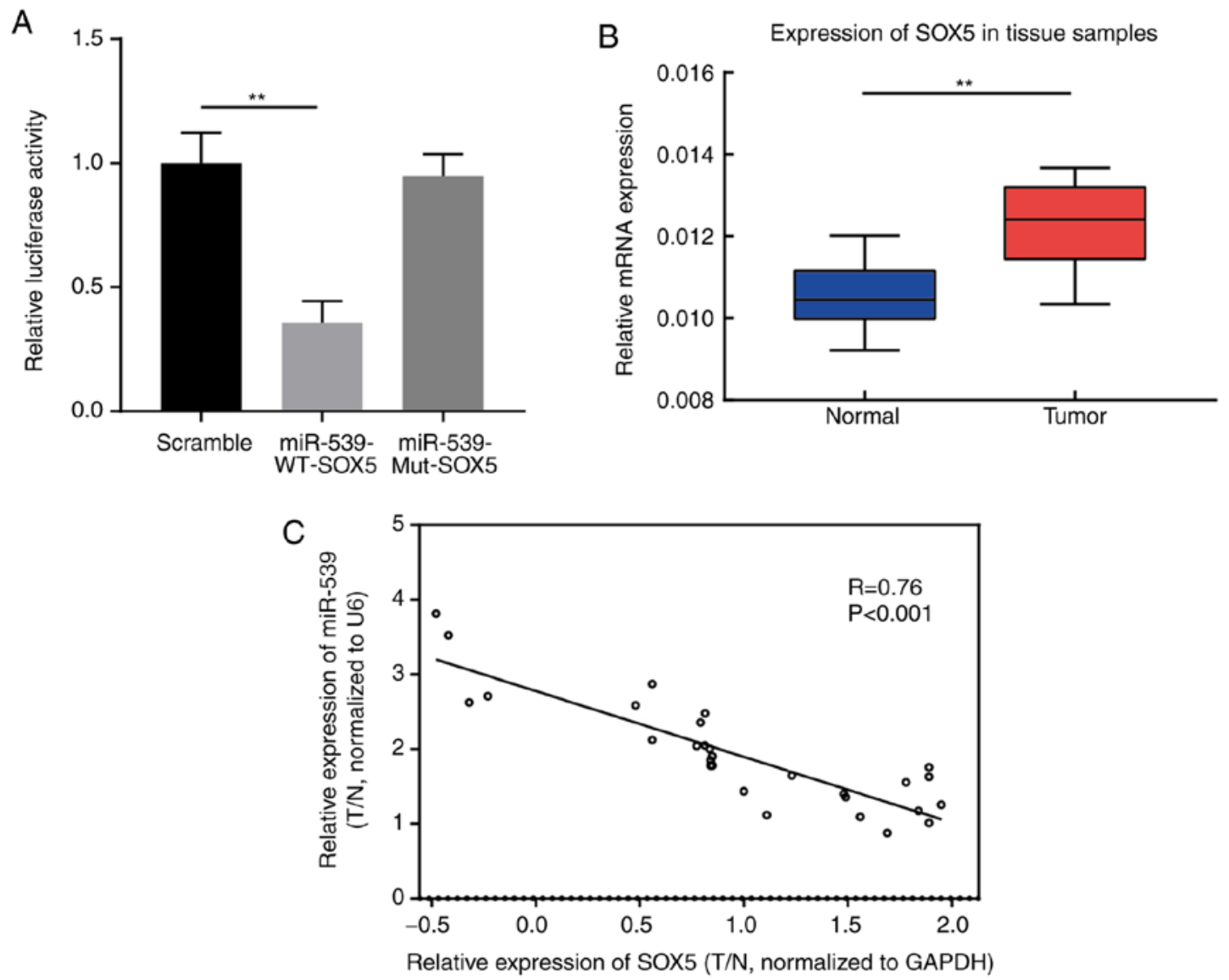

Figure 3. Targeting association between miR-539 and SOX5 gene. (A) Luciferase activity of SOX5 WT and Mut reporter vectors. (B) Expression of SOX5 in tumor and adjacent normal tissues samples $(\mathrm{n}=30)$. (C) Spearman's correlation analysis between miR-539 and SOX5 expression in clinical tissue samples of gastric cancer. ${ }^{* *} \mathrm{P}<0.01$, as indicated. miR, microRNA; SOX5, SRY-box 5; WT, wild type; Mut, mutant; T/N, tumor/normal.

gene overexpression. Therefore, it was hypothesized that the regulation of miR-539 on gastric cancer cells may be achieved by SOX5. Therefore, rescue experiments were conducted to further identify the underlying mechanism. MGC803 and SGC7901 cells were transfected with miR-NC, miR-539 mimic, or co-transfected with miR-539 and SOX5 overexpression vectors. The results of CCK- 8 assay revealed that, compared with the group transfected with miR-539 mimic alone, MGC803 (Fig. 5A) and SGC7901 (Fig. 5B) cells transfected with both miR-539 and SOX5 overexpression vectors exhibited significantly increased proliferation. Furthermore, transwell assay results demonstrated that the migration of MGC803 (Fig. 5C) and SGC7901 (Fig. 5D) cells in miR539 + SOX5 group was also significantly improved. In combination with the previous experimental results, it is confirmed that the regulation of miR-539 on gastric cancer cells was achieved by SOX5.

\section{Discussion}

Gastric cancer is a heterogeneous disease, whose occurrence and development are associated with multiple environmental factors and cancer pathways. The external factors include Helicobacter pylori and Epstein-Barr virus infection, alcohol abuse, body mass index and physical activity (17), while the internal factors are manifested in genetic inheritance (18). In the treatment of early gastric cancer, surgical resection remains the main therapeutic strategy. During the postoperative period, other comprehensive treatment measures, such as adjuvant chemotherapy, can be applied. However, the overall outcome of gastric cancer remains unfavorable. In recent years, molecular targeted therapy has gradually a research focus, and increasing molecular target drugs have been applied in clinical trials. Thus, exploration of new molecular markers or targets will be helpful for diagnosis in gastric cancer.

Accumulating studies have demonstrated that microRNAs can function as oncogenes or tumor suppressor genes by differential expression in a variety of tumor cells. Some microRNAs can promote the proliferation, invasion or metastasis of tumor cells in malignant tumors through multiple pathways. For instance, miR-539 is able to inhibit the invasion and migration of osteogenic sarcoma cells by targeting matrix metalloproteinase-8 (18). In HCC, low expression of miR-539 was reported in cancer tissues and cells, and this miRNA was able to induce HCC cell apoptosis by mediating signal transducer and activator of transcription 3 pathway (11).

In the present study, miR-539 was observed to be downregulated in gastric cancer tissues and cell lines. In combination with the findings of a previous study (13), it is hypothesized that miR-539 functions as a tumor suppressor in gastric cancer. In the subsequent experiments, the proliferation of MGC803 and SGC7901 cells transfected with miR-539 mimic was found to be significantly decreased. The results of transwell assay indicated that miR-539 promoted the migration of gastric cancer 
A

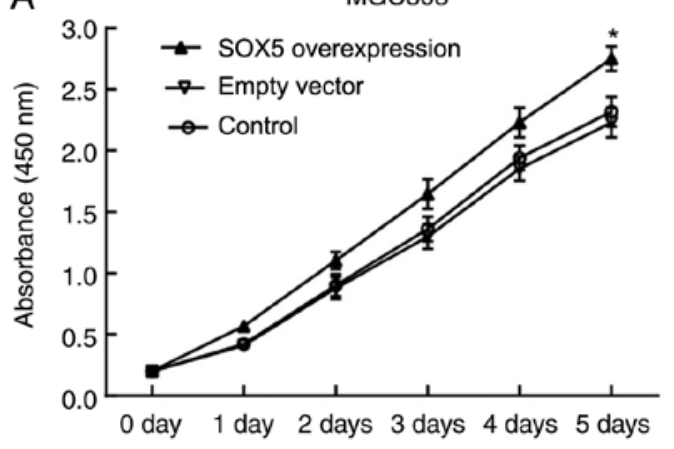

C

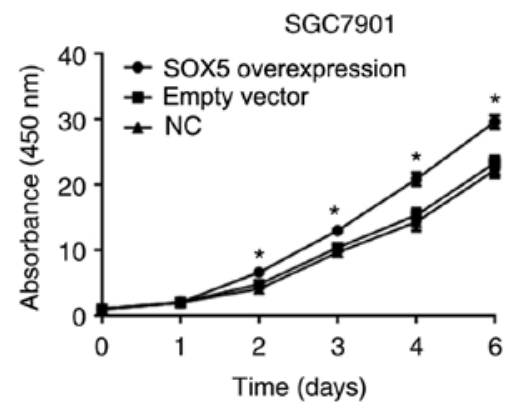

E

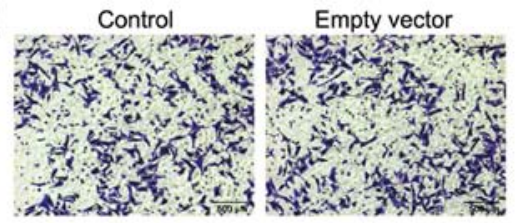

SOX5 overxpression

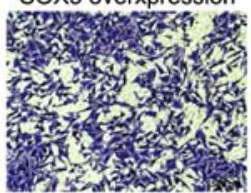

\section{G}
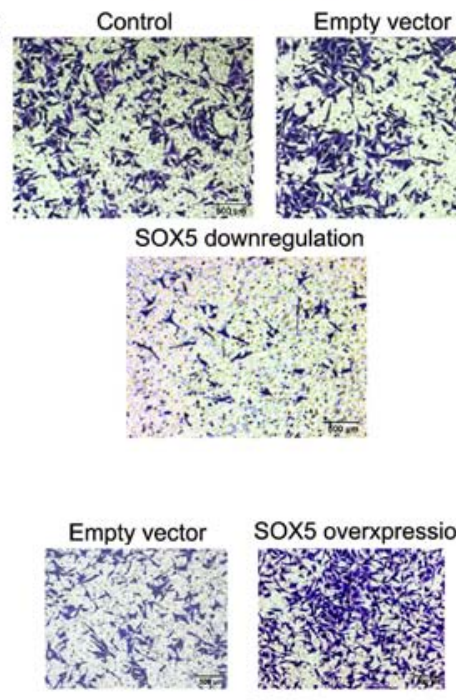

Empty vector

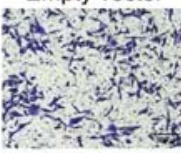

Empty vector

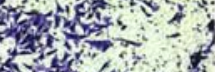

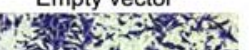
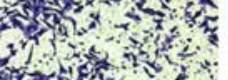

B

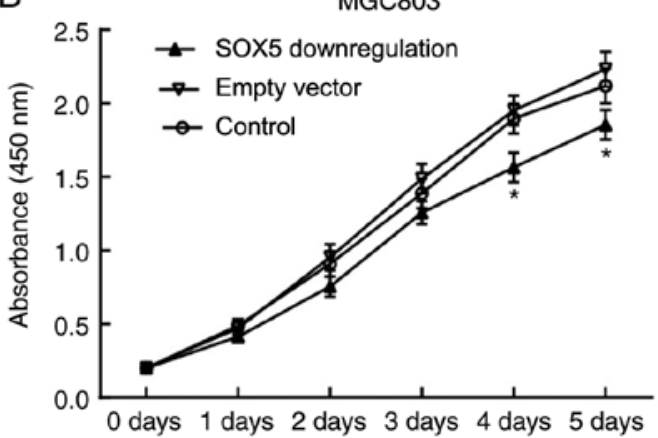

D
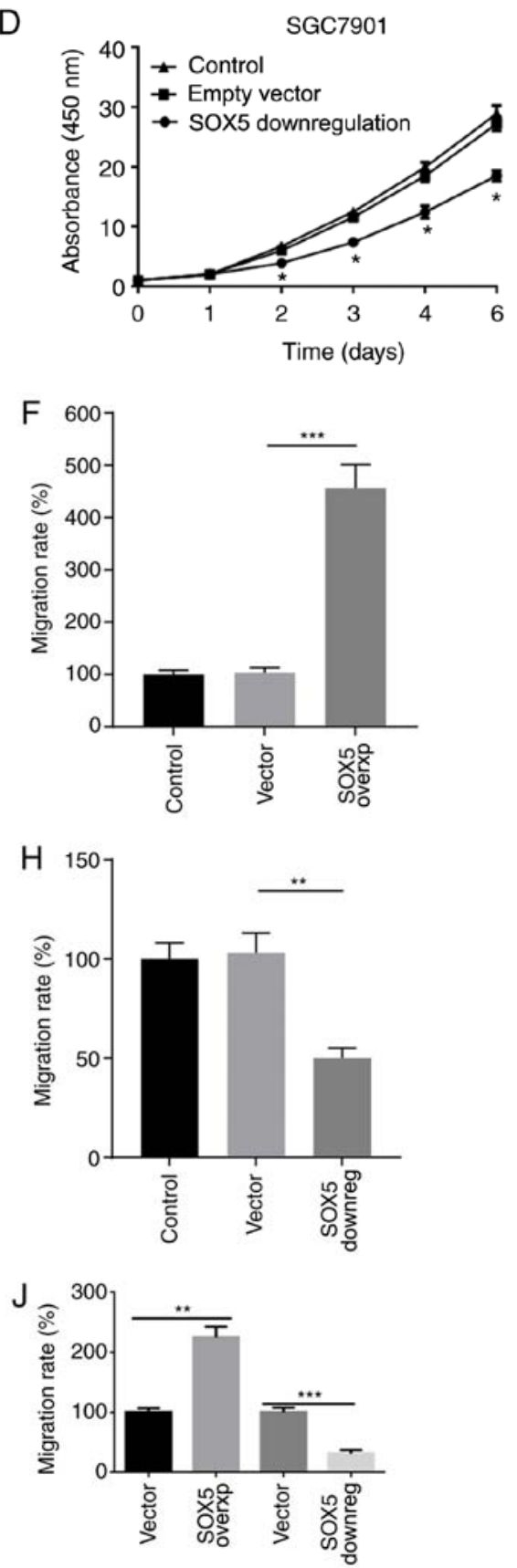

Figure 4. Effect of SOX5 on the proliferation and migration of MGC803 and SGC7901. Effects of (A) overexpression and (B) silencing of SOX5 on the growth of MGC803 cells, and effects of (C) overexpression and (D) silencing of SOX5 on the growth of SGC7901 cells were detected via Cell Counting Kit-8 assay. ${ }^{*} \mathrm{P}<0.05$ vs. control group. (E) Cell images and (F) migration rate examined by transwell assay to determine the effect of SOX5 overexpression in MGC803 cells. (G) Cell images and (H) migration rate examined by transwell assay to determine the effect of SOX5 downregulation in SGC7901 cells. (I) SOX5 overexpression and (J) SOX5 silencing effect on the migration of MGC803 via transwell assays. Scale bar, $500 \mu \mathrm{M}$. ${ }^{* *} \mathrm{P}<0.01$ and ${ }^{* * * *} \mathrm{P}<0.001$, as indicated. SOX5, SRY-box 5. 

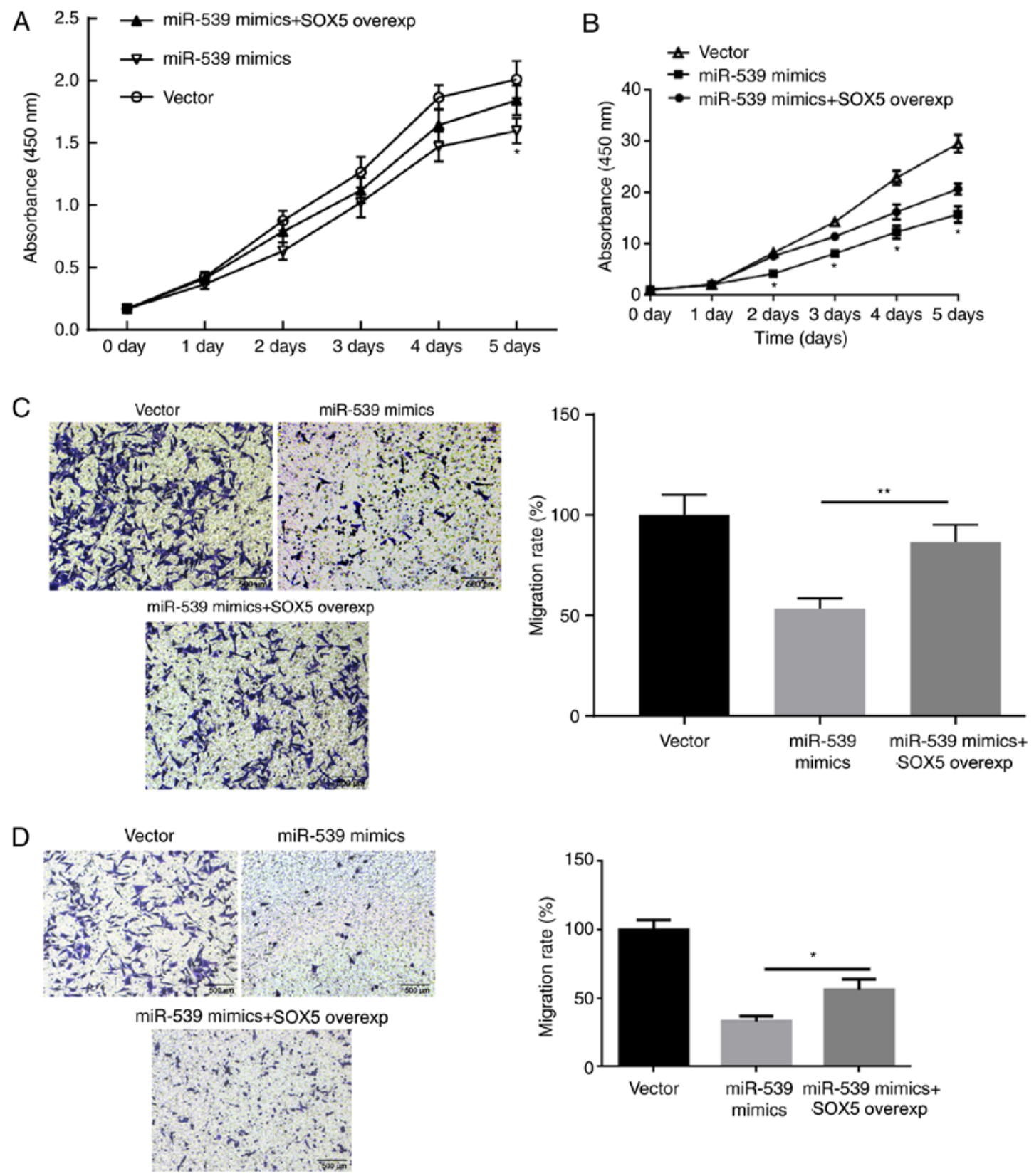

Figure 5. SOX5 restored the effect of miR-539 on the proliferation and migration of gastric cancer cells. Cells were transfected with miR-NC, miR-539 mimic, or co-transfected with both miR-539 and SOX5 overexpression vectors. (A) MGC803 and (B) SGC7901 cell growth was examined following miR-539 and SOX5 vector transfection. Compared with the group transfected with miR-539 mimic alone, cells cotransfected with miR-539 mimic and SOX5 overexpression vector exhibited significantly increased proliferation. "P<0.05 vs. Vector (control) group. (C) MGC803 and (D) SGC7901 cell migration was examined by transwell assay following miR-539 mimic and SOX5 vector transfection (scale bars, $500 \mu \mathrm{m}$ ). Compared with the group transfected with miR-539 mimic alone, cells co-transfected with miR-539 mimic and SOX5 overexpression vectors exhibited significantly increased migration. ${ }^{*} \mathrm{P}<0.05$ and ${ }^{* *} \mathrm{P}<0.01$, as indicated. miR, microRNA; SOX5, SRY-box 5; NC, negative control.

cells. Next, the potential target gene of miR-539 was predicted by TargetScan and miRanda, and it was observed that SOX5 was a potential target of miR-539, which was then verified by luciferase assay. Compared with Mut SOX5 reporter vector, miR-539 mimic significantly reduced the luciferase activity of WT SOX5 reporter vector.

Previous studies have demonstrated that SOX5 participates in the process of tumor epithelial-mesenchymal transition (EMT). EMT is accompanied by marked changes in cell morphology and behavior, such as in cell proliferation, differentiation, adhesion and migration. In addition, EMT can enhance the migration and invasion properties of cancer cells, and induce stem cell properties in tumor cells $(19,20)$. Research has also revealed that SOX5 in breast cancer can directly bind to the Twist 1 promoter to activate the gene expression, while it also controls the ETM process, suggesting that SOX5 participates in the progression of breast cancer by activating the Twist1/ETM axis (21). However, in osteogenic sarcoma, SOX5 promotes cell migration and invasion, which may be achieved by regulating the expression of Snail, so as to promote EMT (22). Consistent with the observations of previous studies investigating SOX5 in other tumors, the present study suggested that SOX5 was highly expressed in clinical cases of gastric 
cancer, and that SOX5 overexpression repressed cell proliferation and migration, which was in contrast to the effect of SOX5 silencing. The results of rescue experiments demonstrated that cell proliferation and migration was significantly increased following transfection with miR-539 and SOX5 overexpression vector, implying that miR-539 inhibited the proliferation and migration of gastric cancer cells by targeting SOX5. Based on the research results, it is speculated that the inhibitory effect of miR-539 on the proliferation and migration of gastric cancer cells may be achieved by indirectly regulating EMT through mediating SOX5 expression.

In conclusion, miR-539 was observed to inhibit the proliferation and migration of gastric cancer cells by targeting SOX5. miR-539 may function as a tumor suppressor and provide a potential target for therapeutic strategies of gastric carcinoma.

\section{Acknowledgements}

Not applicable.

\section{Funding}

The present study was supported by Scientific Research Project of Education Department of Hunan Province (grant no. $18 \mathrm{c} 1220)$.

\section{Availability of data and materials}

All data generated or analyzed during the present study are included in this published article.

\section{Authors' contributions}

YZ conceived and designed the study, and wrote the manuscript. SD performed the expriments and analyzed the data. All authors have read and approved the final manuscript.

\section{Ethics approval and consent to participate}

The present study was approved by the Ethics Committee of Changde Vocational Technical College Research (Changde, China). Patients provided signed informed consent prior to participation in the study.

\section{Patient consent for publication}

Not applicable.

\section{Competing interests}

The authors declare that they have no competing interests.
3. Morgan KA (ed): Current controversies in cancer care for the surgeon. Springer, 2016

4. Soares FA, Coimbra FJ, Pelosof AG, Freitas HC, Begnami MD, Costa Jr WL, Fannelli MF, Lopes de Mello CA, morim M, Pizzi MP, et al: Genomics and epidemiology for gastric adenocarcinomas. Appl Cancer Res 37: 7, 2017.

5. Rugge M, Fassan M and Graham DY: Epidemiology of gastric cancer. Gastric Cancer. Springer, pp23-34, 2015.

6. Esquela-Kerscher A and Slack FJ: Oncomirs-microRNAs with a role in cancer. Nat Rev Cancer 6: 259-269, 2006.

7. Bushati N and Cohen SM: microRNA functions. Ann Rev Cell Dev Bio 23: 175-205, 2007.

8. Bartel DP: MicroRNAs: Genomics, biogenesis, mechanism, and function. Cell 116: 281-297, 2004.

9. Li Q, Li J, Dai W, Li YX and Li YY: Differential regulation analysis reveals dysfunctional regulatory mechanism involving transcription factors and microRNAs in gastric carcinogenesis. Artif Intell Med 77: 12-22, 2017.

10. Mirghasemi A, Taheriazam A, Karbasy SH, Torkaman A, Shakeri M, Yahaghi E and Mokarizadeh A: Retraction Note: Down-regulation of miR-133a and miR-539 are associated with unfavorable prognosis in patients suffering from osteosarcoma. Cancer Cell Int 16: 84, 2016.

11. Zhu C, Zhou R, Zhou Q, Chang Y and Jiang M: microRNA-539 suppresses tumor growth and tumorigenesis and overcomes arsenic trioxide resistance in hepatocellular carcinoma. Life Sci 166: 34-40, 2016.

12. Lv LY, Wang YZ, Zhang Q, Zang HR and Wang XJ: miR-539 induces cell cycle arrest in nasopharyngeal carcinoma by targeting cyclin-dependent kinase 4. Cell Biochem Funct 33: $534-540,2015$

13. Retraction: Xianzheng Gao, Shenglei Li, Wencai Li, Guannan Wang, Wugan Zhao, Jing Han, Changying Diao, Xiaohui Wang, and Mingzhi Zhang,MicroRNA-539 suppresses tumor cell growth by targeting the WNT8B gene in non-small cell lung cancer. J. Cell. Biochem. Accepted Article doi.org/10.1002/jcb.26634. J Cell Biochem: Dec 21, 2017 (Epub ahead of print).

14. Gu L and Sun W: MiR-539 inhibits thyroid cancer cell migration and invasion by directly targeting CARMA1. Biochem Biophys Res Commun 464: 1128-1133, 2015.

15. Chen GD and Liu YL: Clinical, endoscopic and pathologic analysis of 36 cases of early gastric cancer. China J Endosc, 2012 (In Chinese).

16. Livak KJ and Schmittgen TD: Analysis of relative gene expression data using real-time quantitative PCR and the 2(-Delta Delta C(T)) method. Methods 25: 402-408, 2001.

17. Singh S and Jha HC: Status of epstein-barr virus coinfection with Helicobacter pylori in gastric cancer. J Oncol 2017: 3456264, 2017.

18. Jin H and Wang W: MicroRNA-539 suppresses osteosarcoma cell invasion and migration in vitro and targeting Matrix metallopeptidase-8. Int J Clin Exp Pathol 8: 8075-8082, 2015.

19. Thiery JP, Acloque H, Huang RY and Nieto MA: Epithelial-mesenchymal transitions in development and disease. Cell 139: 871-890, 2009.

20. Mani SA, Guo W, Liao MJ, Eaton EN, Ayyanan A, Zhou AY, Brooks M, Reinhard F, Zhang CC, Shipitsin M, et al: The epithelial-mesenchymal transition generates cells with properties of stem cells. Cell 133: 704-715, 2008.

21. Pei XH, Lv XQ and Li HX: Sox5 induces epithelial to mesenchymal transition by transactivation of Twist1. Biochem Biophys Res Commun 446: 322-327, 2014.

22. Zhang D and Liu S: SOX5 promotes epithelial-mesenchymal transition in osteosarcoma via regulation of Snail. J BUON 22: 258-264, 2017.

\section{References}

1. Parkin DM: The global health burden of infection-associated cancers in the year 2002. Int J Cancer 118: 3030-3044, 2006.

2. Siegel R, Ma J, Zou Z and Jemal A: Cancer statistics, 2014. CA Cancer J Clin 64: 9-29, 2014. 